\title{
The degradation of nucleotide triphosphates extracted under boiling ethanol conditions is prevented by the yeast cellular matrix
}

\author{
Andres Gil $^{1}$ - David Siegel ${ }^{1} \cdot$ Silke Bonsing-Vedelaar ${ }^{2} \cdot$ Hjalmar Permentier $^{3}$ • \\ Dirk-Jan Reijngoud $^{4} \cdot$ Frank Dekker $^{5} \cdot$ Rainer Bischoff $^{1}$
}

Received: 14 September 2016/ Accepted: 11 November 2016/Published online: 28 November 2016

(C) The Author(s) 2016. This article is published with open access at Springerlink.com

\begin{abstract}
Introduction Boiling ethanol extraction is a frequently used method for metabolomics studies of biological samples. However, the stability of several central carbon metabolites, including nucleotide triphosphates, and the influence of the cellular matrix on their degradation have not been addressed.

Objectives To study how a complex cellular matrix extracted from yeast (Saccharomyces cerevisiae) may affect the degradation profiles of nucleotide triphosphates extracted under boiling ethanol conditions.

Methods We present a double-labelling LC-MS approach with a ${ }^{13} \mathrm{C}$-labeled yeast cellular extract as complex
\end{abstract}

Electronic supplementary material The online version of this article (doi:10.1007/s11306-016-1140-4) contains supplementary material, which is available to authorized users.

Rainer Bischoff

r.p.h.bischoff@rug.nl

1 Department of Pharmacy, Analytical Biochemistry, University of Groningen, Antonius Deusinglaan 1, Building 3226, Room 601, 9713 AV Groningen, The Netherlands

2 Biomolecular Sciences and Biotechnology Institute, Molecular Systems Biology, University of Groningen, Nijenborgh 4, 9747 AG Groningen, The Netherlands

3 Department of Pharmacy, Interfaculty Mass Spectrometry Center, University of Groningen, Antonius Deusinglaan 1, 9713 AV Groningen, The Netherlands

4 University Medical Center Groningen, Department of Pediatrics, Center for Liver, Digestive and Metabolic Diseases, University of Groningen, PO Box 30.001, 9700 RB Groningen, The Netherlands

5 Department of Pharmacy, Pharmaceutical Gene Modulation, University of Groningen, Antonius Deusinglaan 1, 9713 AV Groningen, The Netherlands surrogate matrix, and ${ }^{13} \mathrm{C}^{15} \mathrm{~N}$-labeled nucleotides as internal standards, to study the effect of the yeast matrix on the degradation of nucleotide triphosphates.

Results While nucleotide triphosphates were degraded to the corresponding diphosphates in pure solutions, degradation was prevented in the presence of the yeast matrix under typical boiling ethanol extraction conditions.

Conclusions Extraction of biological samples under boiling ethanol extraction conditions that rapidly inactivate enzyme activity are suitable for labile central energy metabolites such as nucleotide triphosphates due to the stabilizing effect of the yeast matrix. The basis of this phenomenon requires further study.

Keywords Metabolomics - Mass spectrometry .

Nucleotides $\cdot$ Stability $\cdot$ Boiling ethanol extraction

\section{Introduction}

Nucleotides are central carbon metabolites that are highly susceptible to enzymatic and/or non-enzymatic degradation. Exact knowledge of the concentrations of ATP, ADP and AMP is, for example, critical to calculate the energy charge of a cell which affects overall metabolism. Enzymatic turnover rates of cytosolic ATP and ADP are in the range of $1.5-2.0 \mathrm{mM} \mathrm{s}^{-1}$, and are affected by minimal changes in the cellular environment (Faijes et al. 2007; Villas-Bôas and Bruheim 2007; Vuckovic 2012). Consequently, instantaneous inactivation of enzymatic degradation processes (quenching) during sample preparation is essential to maintain nucleotide concentrations at the original levels (León et al. 2013; Lu et al. 2008; Vuckovic 2012). On the other hand matrix components, such as divalent cations, particularly $\mathrm{Ni}^{2+}, \mathrm{Zn}^{2+}, \mathrm{Mn}^{2+}$ and $\mathrm{Cu}^{2+}$ have been shown to catalyze the non-enzymatic hydrolytic 
degradation of ATP, GTP, ITP, and CTP (Gil et al. 2015). When these nucleotides are incubated in the presence of $\mathrm{Cu}^{2+}$ at $50{ }^{\circ} \mathrm{C}$ and $\mathrm{pH} 5.0-8.0$, there is a decrease in halflives from approximately 10 days to $46.2 \mathrm{~min}$ for ATP, $385 \mathrm{~min}$ for GTP, $770 \mathrm{~min}$ for ITP, and $2310 \mathrm{~min}$ for CTP (Amsler and Sigel 1976; Sigel and Amsler 1976; Sigel and Hofstetter 1983). Thus, besides enzymatic transformation during sample preparation, the hydrolysis of nucleotide triphosphates caused by components of the sample matrix deserves attention, since it may affect the final quantitative results of metabolomics studies.

Several studies dealing with nucleotide stability during sample preparation for metabolomics, have focused on the development of new types of quenching/extraction processes and the evaluation of residual enzymatic activity (Canelas et al. 2008, 2009; Loret et al. 2007; Vuckovic 2012). Despite these efforts, there is a lack of knowledge regarding the changes induced by the physicochemical conditions to which samples are exposed at the processing stage. Usually, at the sample processing stage, working temperatures are in the range of $4-25{ }^{\circ} \mathrm{C}$ for sampling, and -40 to $95{ }^{\circ} \mathrm{C}$ for quenching/extraction, while the $\mathrm{pH}$ can vary between 2 and 8 . Furthermore, sample preparation may take up to $24 \mathrm{~h}$ in chemical environments rich in water and oxygen, as well as in the presence of light (Gil et al. 2015; Siegel et al. 2014). All of these conditions may induce purely chemical degradation processes that might be exacerbated by components of the sample matrix.

Boiling ethanol, first introduced as an approach to extract metabolites from yeast, has become one of the most frequently used methods formetabolomics studiesin otherbiological systems(Airoldietal.2015; DunnandWinder2011; Gonzalezetal. 1997). While several researchers have used this type of extraction with differentmodifications, according toparticularneeds (Buescheretal.2010;Canelasetal.2009;Faijesetal.2007;Paglia et al. 2012; Sellick et al. 2010), there is little information regarding the stability of critical molecules, including nucleotides,undersuchharshextractionconditions. Thisissuehasbeen addressedbyevaluatingthestabilityofthefourmajornucleotide triphosphates present in cells (ATP, GTP, UTP, and CTP) showing that there is significant degradation of these metabolites, caused by the extraction procedure (Giletal.2015).

The purpose of this study was to determine how a complex cellular matrix extracted from yeast (Saccharomyces cerevisiae) may affect the degradation profiles of nucleotides under boiling ethanol conditions. To this end we used a double-labelling approach with ${ }^{13} \mathrm{C}$-labeled yeast extract as complex surrogate matrix and ${ }^{13} \mathrm{C}^{15} \mathrm{~N}$-labeled nucleotides as internal standards. To get a better understanding of the effect of the yeast matrix on nucleotide triphosphate degradation, we evaluated the effect of a range of matrix components on stability.

\section{Materials and methods}

For chemicals, the calibration with ${ }^{13} \mathrm{C}^{15} \mathrm{~N}$-labeled nucleotides, and the statistical analysis see Electronic Supplementary Material.

\subsection{Generation of ${ }^{13} \mathrm{C}$-labeled yeast cellular matrix}

Preparation of ${ }^{13} \mathrm{C}$-labeled yeast cell extracts was done according to Buescher et al. (2010), with slight modifications. S. cerevisiae, strain YSBN6 (MATa; genotype: FY3 ho::HphMX4 derived from the S288C parental strain) was grown on glucose minimal medium (Verduyn medium)

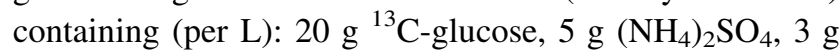
$\mathrm{KH}_{2} \mathrm{PO}_{4}, \quad 0.5 \mathrm{~g} \quad \mathrm{MgSO}_{4} \cdot 7 \mathrm{H}_{2} \mathrm{O}, 4.5 \mathrm{mg} \quad \mathrm{ZnSO}_{4} \cdot 7 \mathrm{H}_{2} \mathrm{O}$, $4.5 \mathrm{mg} \mathrm{CaCl} 2 \cdot 2 \mathrm{H}_{2} \mathrm{O}, 3 \mathrm{mg} \mathrm{FeSO} \cdot 7 \cdot 7 \mathrm{H}_{2} \mathrm{O}, 2.5 \mathrm{mg}$ inositol,

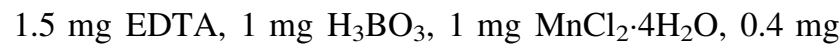
$\mathrm{NaMoO}_{4} \cdot 2 \mathrm{H}_{2} \mathrm{O}, 0.3 \mathrm{mg} \mathrm{CoCl} \cdot 6 \mathrm{H}_{2} \mathrm{O}, 0.3 \mathrm{mg} \mathrm{CuSO} \cdot \cdot 5 \mathrm{H}_{2}$ $\mathrm{O}, 100 \mu \mathrm{g} \mathrm{KI}, 100 \mu \mathrm{g}$ thiamine, $100 \mu \mathrm{g}$ Ca-pantothenic acid, $100 \mu \mathrm{g}$ nicotinic acid, $100 \mu \mathrm{g}$ pyridoxine, $20 \mu \mathrm{g}$ p-aminobenzoic acid, and $5 \mu \mathrm{g}$ biotin, buffered with $10 \mathrm{mM}$ potassium phthalate to $\mathrm{pH} 5.0$ (Verduyn et al. 1992). Cultures of $12 \times 85 \mathrm{~mL}$ were incubated in $1 \mathrm{~L}$ flasks at $30{ }^{\circ} \mathrm{C}$ with shaking at $250 \mathrm{rpm}$ and grown to $\mathrm{OD}_{600}=3.5 \pm 0.2 .0 .213 \mathrm{~g}$ of ${ }^{13} \mathrm{C}$-labelled glucose was added $10 \mathrm{~min}$ prior to harvesting. For harvesting, $80 \mathrm{~mL}$ of culture were mixed with $320 \mathrm{~mL}$ of methanol, precooled to $-80{ }^{\circ} \mathrm{C}$. The methanol-culture mix was kept below $-40{ }^{\circ} \mathrm{C}$ in a cold ethanol/ethylene glycol bath $(1: 1 \mathrm{mix})$ cooled with dry ice. Cells were separated by centrifugation at $-9{ }^{\circ} \mathrm{C}$ and $2500 \mathrm{~g}$ for $15 \mathrm{~min}$ and the pellet was kept cold in dry ice prior to extraction.

Extraction was done as previously described (Buescher et al. 2010) with slight modifications. Briefly, samples were extracted twice with $350 \mathrm{~mL}$ of $60 \%$ (aq) ethanol for $2 \mathrm{~min}$ at $78{ }^{\circ} \mathrm{C}$. After each extraction step, the mixture was centrifuged for 2 min at $13,000 \times g$ and $4{ }^{\circ} \mathrm{C}$ by means of a JLA-10.500 fixed angle rotor in a Beckman Coulter J2 centrifuge (Beckman Coulter, Inc., USA) and the supernatant was collected. The pooled supernatants were frozen in liquid nitrogen and the solvent removed in a $\mathrm{VaCo} 2$ freeze dryer (Zirbus Technology, Bad Grund, Germany). The residue was resuspended in extraction solvent (ethanol $60 \%$ aq) and the resulting solution was aliquoted and stored at $-80{ }^{\circ} \mathrm{C}$ until further use.

\subsection{In-house production of ${ }^{13} \mathrm{C}^{15} \mathrm{~N}$-labeled nucleotide di- and monophosphates}

Labelled nucleotide di- and monophosphate internal standards were produced by a controlled degradation of ${ }^{13} \mathrm{C}^{15} \mathrm{~N}$-labeled ATP, GTP, CTP and UTP. Briefly, $800 \mu \mathrm{L}$ 
of $75 \%$ (aq) ethanol preheated at $95{ }^{\circ} \mathrm{C}$ were added to $200 \mu \mathrm{L}$ of ${ }^{13} \mathrm{C}^{15} \mathrm{~N}$-labeled nucleotide standard solutions at $100 \mathrm{mM}$. The mixture was transferred to $1.5 \mathrm{~mL}$ vials and incubated at $95{ }^{\circ} \mathrm{C}$ for 130,200 and $220 \mathrm{~min}$ for ATP/GTP, CTP and UTP, respectively, since at these time points similar concentrations of the original compounds and the main degradation products (i.e. the di- and monophosphates) were obtained (Fig. 1). Reactions were stopped by snap-freezing in liquid nitrogen. The vials were thawed and excess solvent evaporated under a stream of nitrogen at room temperature. The residues were resuspended in $1 \mathrm{~mL}$ of ultrapure water and mixed to form a master solution of ${ }^{13} \mathrm{C}^{15} \mathrm{~N}$-labeled nucleotides (tri-, di- and monophosphates) that was used as an internal standard for quantitative purposes in the remaining experimental work. Extracted-ion chromatograms (EIC) for the ${ }^{13} \mathrm{C}^{15} \mathrm{~N}$-labeled tri-, di-, and monophosphates were compared with commercially available unlabeled compounds by detecting their respective exact mass-to-charge ratios $(\mathrm{m} / \mathrm{z})$ in high-resolution mode with an extraction window of $5 \mathrm{ppm}$. The internal standards showed identical MS/MS spectra (except for the mass shift) and retention times as the unlabeled nucleotides (ATP, ADP, AMP, GTP, GDP, GMP, CTP, CDP, CMP, UTP, UDP and UMP) (Fig. S1).

\subsection{LC-MS}

Nucleotides were separated in the HILIC mode using a Luna $\mathrm{NH}_{2}$ column $(3 \mu \mathrm{m}, 100 \times 2 \mathrm{~mm}$; Phenomenex $)$ on an Acquity UPLC system (Waters, Manchester, UK). Mobile phases consisted of a mixture of $5 \mathrm{mM}$ ammonium acetate in water at $\mathrm{pH} 9.9$ (eluent $\mathrm{A}$ ) and acetonitrile (eluent B). Linear gradient elution went from $30 \%$ eluent A to $99 \%$ eluent $\mathrm{A}$ in $8 \mathrm{~min}$, followed by isocratic elution at 99\% eluent A until $14 \mathrm{~min}$. A conditioning cycle of $6 \mathrm{~min}$ with the initial proportions of eluents $\mathrm{A}$ and $\mathrm{B}$ was performed prior to the next analysis. The column temperature was set at $20^{\circ} \mathrm{C}$, the flow rate was $0.25 \mathrm{~mL} / \mathrm{min}$, and the injection volume per sample was $10 \mu \mathrm{L}$.

Mass spectrometry detection was performed using a Waters Synapt G2-Si high-resolution Q-TOF mass spectrometer. The analytes (nucleotide triphosphates) and their respective degradation products (nucleotide $\mathrm{di}^{-}$and monophosphates) were detected by electrospray ionization in negative mode $\left(\mathrm{ESI}^{-}\right)$. Nitrogen and argon were used as desolvation and collision gas, respectively. Data were acquired over the $\mathrm{m} / \mathrm{z}$ range of $50-1200 \mathrm{Da}$, with the source temperature set at $150{ }^{\circ} \mathrm{C}$, desolvation temperature at $400{ }^{\circ} \mathrm{C}$, cone voltage at $30 \mathrm{~V}$ and capillary voltage at $2000 \mathrm{~V} . \mathrm{MS}^{\mathrm{E}}$ data acquisition was used in centroid and sensitivity modes. The collision energy potential setting was $2 \mathrm{~V}$ for the low-collision energy scan, and 10-30 V for the high-collision energy scan. As a result, for each sample, both precursor ion and fragment ion information was obtained from a single LC injection. The system was equipped with an integral LockSpray unit with its own reference sprayer that was controlled automatically by the acquisition software to collect a reference scan every $10 \mathrm{~s}$ lasting $0.3 \mathrm{~s}$. The LockSpray internal reference used for these experiments was a $0.2 \mathrm{ng} / \mu \mathrm{L}$ leucine-enkephalin solution $(\mathrm{m} / \mathrm{z} 554.2615$ in negative ion electrospray mode) infused at $10 \mu \mathrm{L} / \mathrm{min}$. Separation (UPLC) and detection
Fig. 1 Kinetic profile of nucleotide triphosphate degradation in $75 \%$ (aq.) boiling ethanol. a ATP, b GTP, c UTP and d CTP. Each time point represents the mean of four independently prepared samples $( \pm \mathrm{SD})$
(A)

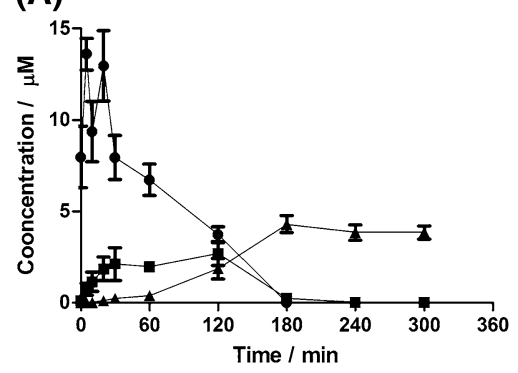

(C)

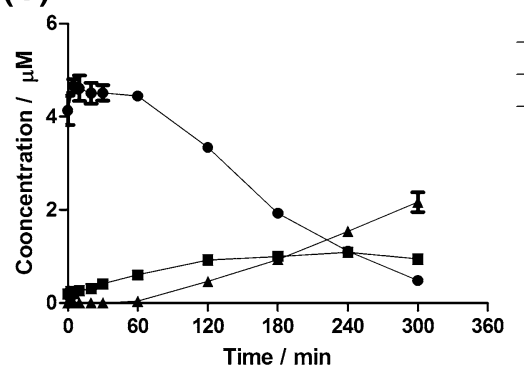

(B)
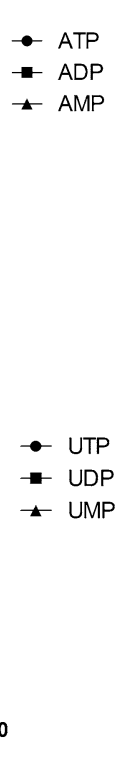

(D)
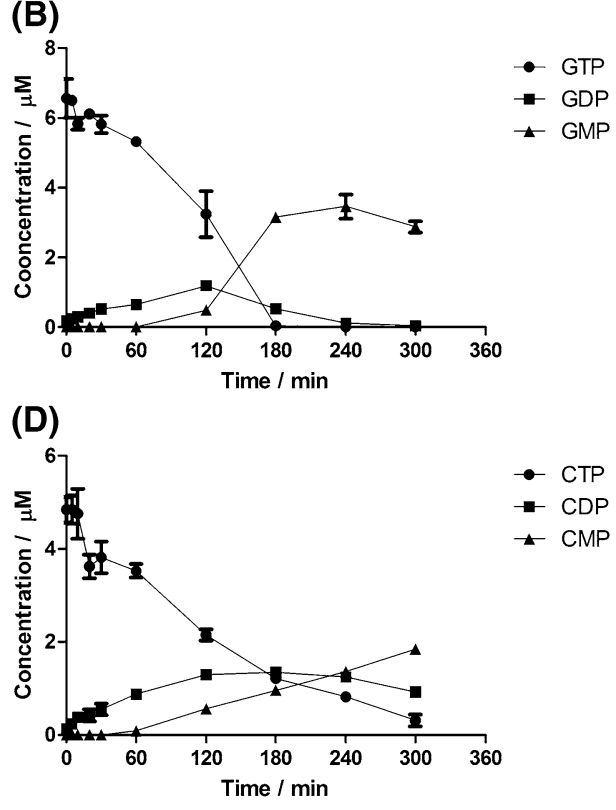
(MS) systems were controlled by MassLynx 4.1 software (Waters, Manchester, UK).

\subsection{Degradation/interconversion of nucleotide triphosphates}

The kinetic profiles of the degradation/interconversion for pure solutions of ATP, GTP, CTP and UTP were assessed under boiling ethanol extraction conditions as used for sample preparation in metabolomics studies. Briefly, tubes containing $496 \mu \mathrm{L}$ of $75 \%$ (aq) ethanol were preheated at $95{ }^{\circ} \mathrm{C}$ for $5 \mathrm{~min}$, followed by the addition of $4 \mu \mathrm{L}$ of each nucleotide standard solution $(500 \mu \mathrm{M})$ and vigorous mixing. To follow the kinetics of the degradation reactions the mixture was incubated at $95{ }^{\circ} \mathrm{C}$ under shaking for $0,3,6,9,12$, and $15 \mathrm{~min}$. Reactions were stopped by snap-freezing in liquid nitrogen and samples were stored on dry ice. Subsequently, samples were thawed and $20 \mu \mathrm{L}$ of the ${ }^{13} \mathrm{C}^{15} \mathrm{~N}$-labeled internal standard solution were added for quantitative analysis. Excess solvent was evaporated under a stream of nitrogen without heating. Finally, samples were reconstituted in $200 \mu \mathrm{L}$ of acetonitrile-water $70: 30$ and stored at $-40{ }^{\circ} \mathrm{C}$ until analysis by LC-MS. The influence of a yeast cellular matrix on the degradation/interconversion of nucleotides was assessed by adding $70 \mu \mathrm{L}$ of the ${ }^{13} \mathrm{C}$-labeled matrix to the reaction mixture and continuing the experimental procedure as described above.

\subsection{Evaluation of selected matrix components on the degradation/interconversion of nucleotide triphosphates}

To study the impact of components usually found in the yeast matrix, the effect of the whole culture medium (carbon source, vitamins, macro and micro elements, etc.) and the influence of particular groups of compounds/ metabolites on the degradation/interconversion of nucleotide triphosphates during boiling ethanol extraction was assessed (Table 1). Briefly, the experimental procedure described in Sect. 2.4 was repeated, but instead of the ${ }^{13} \mathrm{C}$ labeled yeast cellular matrix, $70 \mu \mathrm{L}$ of Verduyn culture medium or $4 \mu \mathrm{L}$ of solutions " $\mathrm{A}$ " to " $\mathrm{H}$ " (Table 1) were added to the reaction mixture. After the experimental procedure the final concentration of all components was $10 \mu \mathrm{M}$ in a volume of $200 \mu \mathrm{L}$. Compounds to be tested were divided into eight groups containing approximately ten compounds each, mainly comprising major central carbon metabolites including amino acids, organic acids, sugar phosphates, coenzymes, etc. (Table 1). Compounds with identical masses were assigned to different groups, so that no group contained two metabolites with masses closer than $2 \mathrm{Da}$, thus avoiding overlapping isotopic peaks.

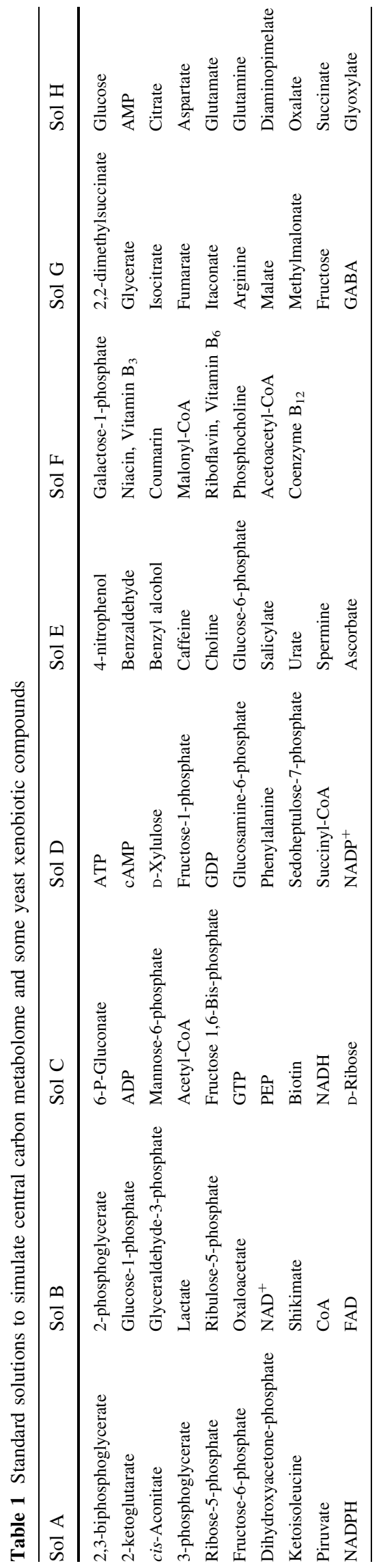




\section{Results and discussion}

\subsection{Production of ${ }^{13} \mathrm{C}^{15} \mathrm{~N}$-labeled nucleotide di- and monophosphates}

Quantitative mass spectrometry in highly complex biological matrices necessitates the use of stable-isotope-labeled internal standards, since ion suppression effects may affect quantification (Wu et al. 2005). While isotopically labeled nucleotide triphosphates $\left({ }^{13} \mathrm{C}^{15} \mathrm{~N}\right.$-labeled ATP, GTP, CTP and UTP) are commercially available, the respective ${ }^{13} \mathrm{C}^{15} \mathrm{~N}$-labeled di- and monophosphates have to be prepared. To generate the missing standards, we subjected nucleotide triphosphates to controlled degradation at $95{ }^{\circ} \mathrm{C}$ for 130, 200 and $220 \mathrm{~min}$ for ATP/GTP, CTP and UTP, respectively, to reach comparable concentrations for each nucleotide (Fig. 1). Comparison of EICs with commercially available unlabeled compounds by LC-MS in high-resolution mode confirmed their identity (Fig. S1).

\subsection{LC-MS method performance and validation}

Chromatographic conditions were optimized on the basis of peak resolution, baseline drift, and retention time resulting in separation of the 12 nucleotide phosphates into 3 groups in $10 \mathrm{~min}$ based on the number of phosphate groups in the hydrophilic interaction liquid chromatography (HILIC) mode at pH 9.9 (Fig. S1). The first group of eluting nucleotides consisted of the monophosphates followed by the di- and triphosphates, respectively. Similar separation patterns have been reported using ion-pairing chromatography albeit with longer retention times (Coulier et al. 2006) or less efficient separation (Seifar et al. 2009).

Quantitative isotope-dilution LC-MS analyses were performed with ${ }^{13} \mathrm{C}^{15} \mathrm{~N}$-labeled nucleotide tri-, di- and monophosphates as internal standards. Calibration curves were obtained by plotting peak area ratios (unlabeled $/{ }^{13-}$ $\mathrm{C}^{15} \mathrm{~N}$-labeled nucleotides) against concentrations of the added unlabeled nucleotides (Figs. S2, S3). Method validation was performed by evaluating linearity (Table S1; $r^{2} \geq 0.9962$ ), intra-day variability (repeatability), inter-day variability (intermediate precision) and accuracy. The limit of quantification (LOQ) was set to $9.8 \mathrm{nM}$, the lowest point on the calibration curve where the analyte responses were at least 5-times higher than the blank and where the coefficient of variation (CV) was less than $20 \%$ in accordance with international guidelines (EMA 2012; HSS-FDA 2013). Repeatability was assessed by testing 5 independently prepared samples that were spiked with each nucleotide at $0.625 \mu \mathrm{M}$ and measured 8 times in one batch. Similar samples were prepared and measured on three different days to assess intermediate precision and accuracy. For all the nucleotides measured in this study, $\mathrm{CV}$ was lower than $\pm 15 \%$ and accuracy higher than $95 \%$, satisfying validation criteria for repeatability, intermediate precision and accuracy (Table S1). The validation results established the suitability of the method for the evaluation of nucleotide degradation under different conditions.

\subsection{Effect of the extraction procedure on the stability of nucleotides}

Whereas enzymatic conversion of metabolites has been studied in great detail, nonenzymatic, chemical conversions have received comparatively little attention. By assessing the stability of nucleotide triphosphates under the conditions of a boiling ethanol extraction, one of the most frequently used methodologies for metabolomics studies, we specifically asked the question whether degradation may be affected by other components in the sample matrix. Extracting pure solutions of the nucleotide triphosphates with ethanol at $95{ }^{\circ} \mathrm{C}$ resulted in the degradation of purine and pyrimidine nucleotide triphosphates with a steady, time-dependent increase in the concentration of ADP and GDP (Fig. 2a, c) and of UDP and CDP (Fig. 3a, c). ADP was increased by $80.6 \%(0.473$ to $0.854 \mu \mathrm{M})$, GDP by $63.4 \%(0.614$ to $1.003 \mu \mathrm{M})$, UDP by $54.6 \%$ (0.341 to $0.526 \mu \mathrm{M})$ and CDP by $64.9 \%(0.477$ to $0.762 \mu \mathrm{M})$ after 15 min incubation $(p<0.05$; ANOVA). While there was a trend towards decreasing concentrations for the nucleotide triphosphates (Figs. 2, 3), these changes were not significant $(p>0.05$; ANOVA). Therefore, changes in the concentration of nucleotide diphosphates are more sensitive indicators of nucleotide triphosphate hydrolysis. Although the concentration of nucleotide monophosphates (AMP, GMP, CMP and UMP) showed a tendency to increase as well, these increases were not statistically significant $(p>0.05$; ANOVA) after 15 min (Figs. 2a, c, 3a, c). However, a significant increase in the concentration of the monophosphates was seen after $60 \mathrm{~min}$ at $95{ }^{\circ} \mathrm{C}$ in boiling ethanol (Fig. 1). According to the exhaustive degradation profiles shown in Fig. 1, in order to see a clear and statistically significant $(p<0.05)$ decrease in the concentration of nucleotide triphosphates and a corresponding increase in the concentration of nucleotide di- and monophosphates, the duration of the experiment at $95{ }^{\circ} \mathrm{C}$ should be extended well beyond $15 \mathrm{~min}$. Even though time and temperature are the most common variables when conducting an extraction of samples with boiling ethanol for metabolomics studies, the temperature range generally applied with this extraction approach lies between 78 and $95{ }^{\circ} \mathrm{C}$, and the time of extraction usually ranges from 1 to 15 min (Faijes et al. 2007; Canelas et al. 2009; Buescher et al. 2010; Sellick et al. 2010; Paglia et al. 2012 and Gil et al. 2015). Therefore, the lack of correlation between the 
Fig. 2 The yeast cellular matrix prevents degradation of purine nucleotide triphosphates during extraction in $75 \%$ (aq.) boiling ethanol. a ATP and c GTP extractions in the absence of the yeast cellular matrix. b ATP and d GTP extractions in the presence of the ${ }^{13} \mathrm{C}$-labeled yeast cellular matrix. Each time point represents the mean of four independently prepared samples $( \pm \mathrm{SD})$

Fig. 3 The yeast cellular matrix prevents degradation of pyrimidine nucleotide triphosphates during extraction in $75 \%$ (aq.) boiling ethanol. a UTP and c CTP extractions in the absence of the yeast cellular matrix. b UTP and d CTP extractions in the presence of the ${ }^{13} \mathrm{C}$-labeled yeast cellular matrix. Each time point represents the mean of four independently prepared samples $( \pm \mathrm{SD})$

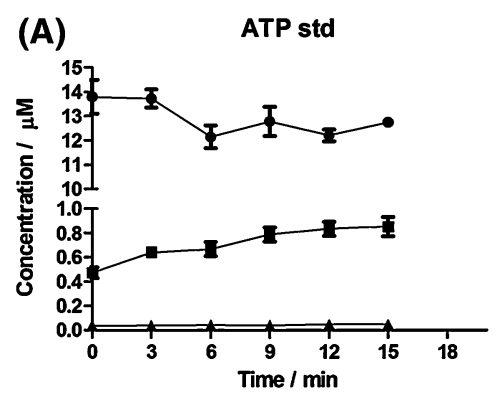

(B)

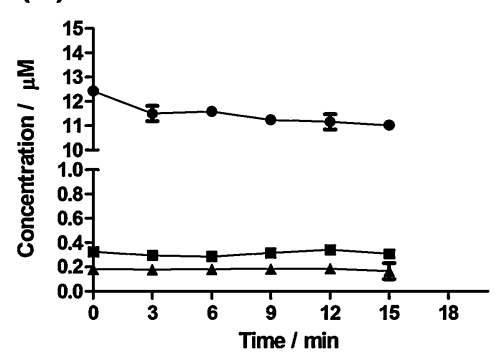

(C)

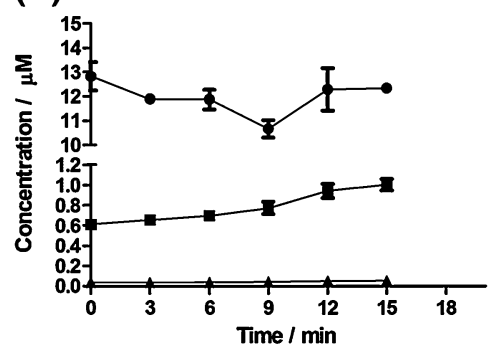

(D)

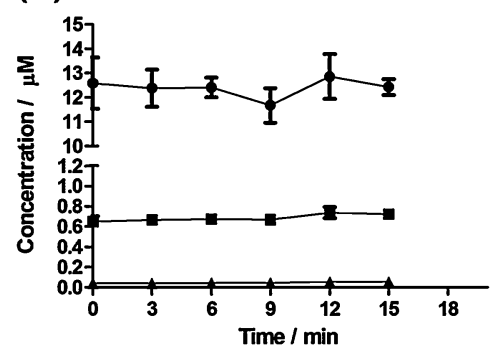

- GTP

- GDP

- GMP
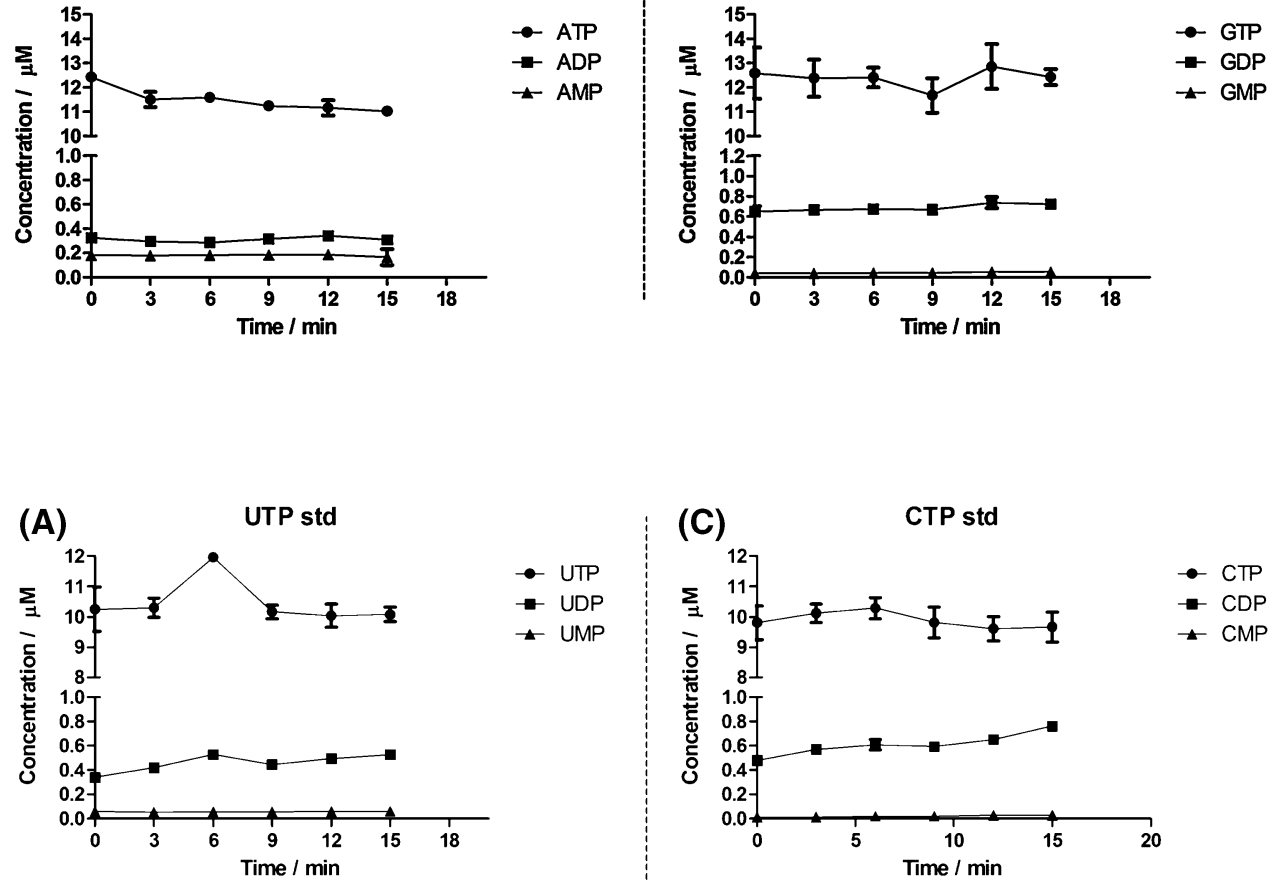

(B)

UTP+M

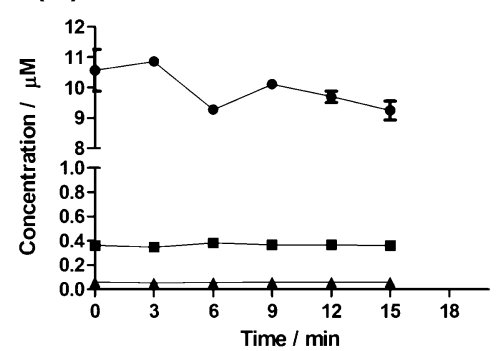

(D)

CTP+M

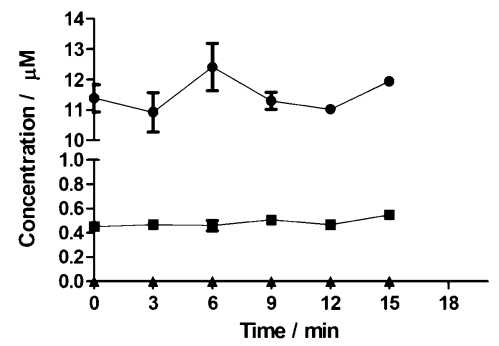

decrease in concentration of nucleotide triphosphates and the increase in nucleotide di- and monophosphates may be due to the limited time of thermal exposure. Our results are consistent with a report by Daniel et al. (2004), who established the degradation kinetics for ATP, ADP and AMP and found similar kinetic profiles for degradation under aqueous conditions for $180 \mathrm{~min}$ at $95{ }^{\circ} \mathrm{C}$. These authors also measured ATP stability in $90 \%$ (aq.) glycerol, an environment more similar to the $75 \%$ (aq.) ethanol that we used, and found them to be similar. It is important to note that an increase in the concentration of one metabolite due to the degradation of another metabolite (interconversion) is not corrected for by stable isotope dilution mass spectrometry, which can only compensate for decreases. This is a limitation of the stable isotope dilution approach in metabolomics. 

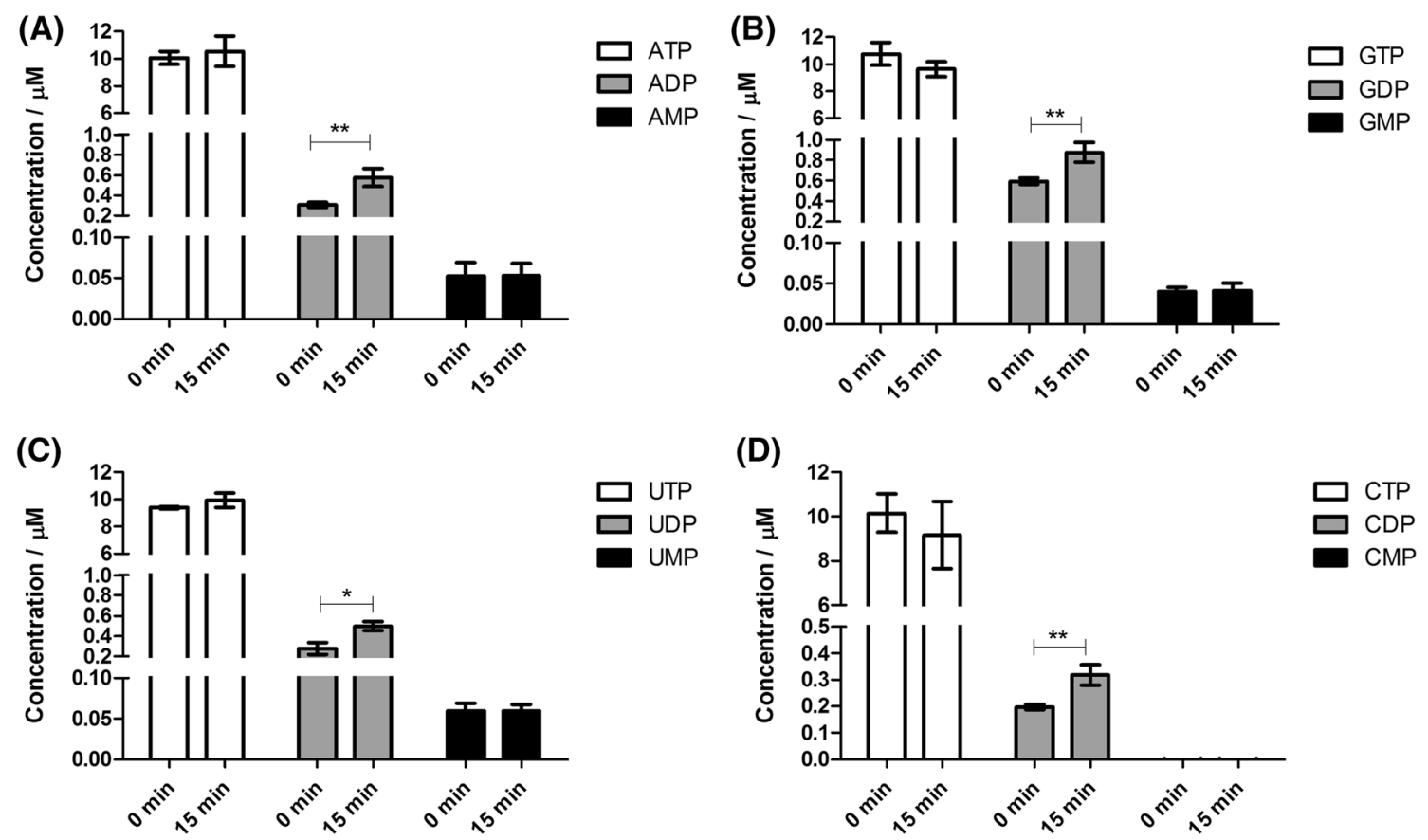

Fig. 4 Effect of the culture medium on the degradation of nucleotide triphosphates. a ATP, b GTP, c UTP, and d CTP were incubated with Verduyn culture medium and extracted with $75 \%$ (aq.) boiling

ethanol. Each bar represents the mean of four independently prepared samples $( \pm \mathrm{SD})$. ANOVA: $* p<0.05 ; * * p<0.01$ and $* * * p<0.001$

\subsection{Effect of a complex cellular matrix on the stability of nucleotides}

Since pure nucleotide triphosphates were degraded when extracted with boiling ethanol, we studied how a complex cellular matrix extracted from yeast (S. cerevisiae) may affect degradation kinetics using a uniformly ${ }^{13} \mathrm{C}$-labeled yeast extract as surrogate matrix and ${ }^{13} \mathrm{C}^{15} \mathrm{~N}$-labeled nucleotides as internal standards. Incubation of unlabeled ATP, GTP, UTP and CTP with the ${ }^{13} \mathrm{C}$-labeled yeast matrix at $95{ }^{\circ} \mathrm{C}$ for 15 min showed a significant decrease in the degradation kinetics indicating a stabilizing effect of the matrix (Figs. 2b, d, 3b, d). In fact, the concentration of ADP, GDP, UDP and CDP as well as that of AMP, GMP, UMP and CMP remained constant during the $15 \mathrm{~min}$ incubation $(p>0.05$; ANOVA). Next to the stabilizing effect of the yeast matrix, we found that the concentration of ADP was decreased at $\mathrm{t}=0 \min (0.473$ to $0.325 \mu \mathrm{M}$; $-68.6 \%)$ and that of AMP was increased (0.032 to $0.182 \mu \mathrm{M} ;+568.7 \%$ ) in comparison to the initial concentration in the absence of the yeast matrix (Fig. 2a, b). While the increase in AMP may be explained by the conversion of other metabolites in the yeast matrix generating AMP (see next section), it is currently unclear how to explain the decrease in ADP concentration.

Since metabolite extractions with boiling ethanol are generally performed without the addition of buffers

(Canelas et al. 2009; Faijes et al. 2007; Paglia et al. 2012; Sellick et al. 2010), we did not buffer the pure nucleotide solutions for our studies. However, trying to explain our results, we measured the $\mathrm{pH}$ of samples with and without the addition of the ${ }^{13} \mathrm{C}$-labeled yeast extract but found no difference $(\mathrm{pH}$ range $=5.8-6.0)$, indicating that stabilization of nucleotide triphosphates was not due to a $\mathrm{pH}$ effect. Earlier Gonzalez et al. (1997) suggested that differential metabolite extraction yields, particularly for ATP and related compounds, may be related to either compartmentalization and/or tight association with macromolecules rather than to their inherent stability in the extracting agents. It is unlikely that such effects play a role during the extraction with boiling ethanol.

\subsection{Influence of the culture medium on the stabilization/degradation of nucleotides}

To discriminate between the stabilizing effect of the yeast matrix and that of the Verduyn culture medium on nucleotide triphosphates, we first evaluated the effect of the culture medium. The Verduyn culture medium is a mixture of organic compounds including glucose, a set of vitamins and a number of salts comprising divalent transition metal cations like $\mathrm{Zn}^{2+}, \mathrm{Mn}^{2+}$ and $\mathrm{Cu}^{2+}$, which have been shown to induce nucleotide degradation (Amsler and Sigel 1976; Sigel and Amsler 1976; Sigel and Hofstetter 1983; 
Verduyn et al. 1992). This effect may, however, be counteracted by the presence of chelating agents such as EDTA in the medium. Overall we found no stabilizing effect, since concentrations of nucleotide diphosphates increased significantly ( $p<0.05$; ANOVA) from 0.309 to $0.578 \mu \mathrm{M}$ (87.1\%) for ADP, 0.597 to $0.879 \mu \mathrm{M}(47.2 \%)$ for GDP, 0.280 to $0.435 \mu \mathrm{M}(55.3 \%)$ for UDP and 0.198 to 0.318 (60.6\%) for CDP during boiling ethanol extraction (Fig. 4).

\subsection{Effect of yeast central carbon metabolites and some xenobiotic compounds on the stabilization/degradation of nucleotides}

We further investigated a range of central carbon metabolites as potential stabilizing factors including amino acids, organic acids, sugar phosphates, coenzymes, and other nucleotides that are typically found in the yeast matrix, as well as some yeast xenobiotic compounds (solutions $\mathrm{A}-\mathrm{H}$ in Table 1). While the concentration of nucleotide triphosphates did not change significantly in the presence of these groups of compounds for the majority of cases (Fig. 5), solutions $\mathrm{C}$ and E for ATP, solutions A and D for UTP, and solution G for CTP showed a decrease in the concentration of the triphosphates after $15 \mathrm{~min}$ of extraction at $95{ }^{\circ} \mathrm{C}$ in boiling ethanol (Fig. 5). In comparison, concentrations of the diphosphates and most of the monophosphates increased indicating that these groups of compounds were not related to the stabilizing effect of the yeast matrix ( $p<0.001$; ANOVA; Fig. 5).

The increase in concentration of nucleotide diphosphates was differentially affected by the tested groups of compounds. The increase for ADP ranged from $18 \%$ in solution C to $272 \%$ in solution D, for GDP from $23 \%$ in solution D to $103 \%$ in solution $\mathrm{F}$, for UDP from $17 \%$ in solution A to $95 \%$ in solution $\mathrm{H}$, and for CDP from $35 \%$ in solution $G$ to $119 \%$ in solution $F$. The increase in
(A)

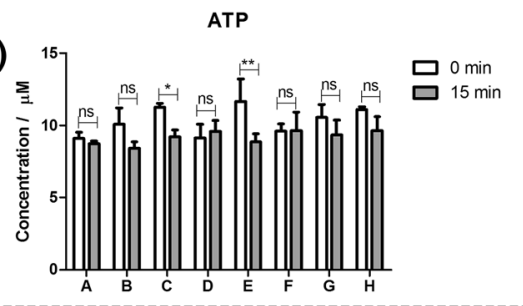

GTP

(B)

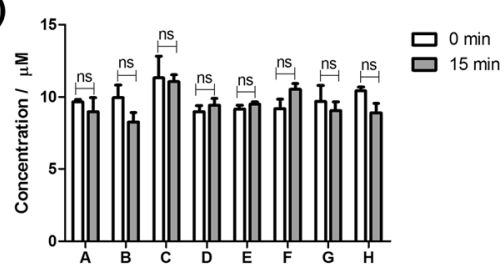

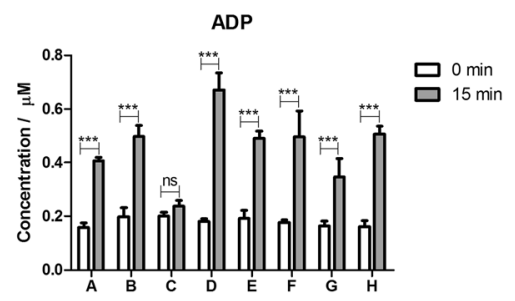

GDP

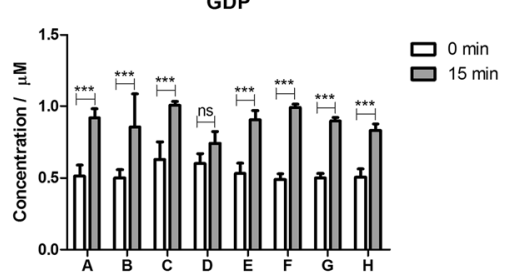

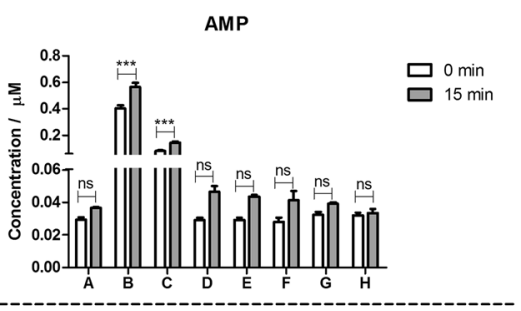

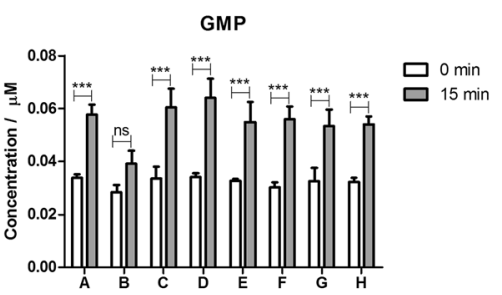

(C)

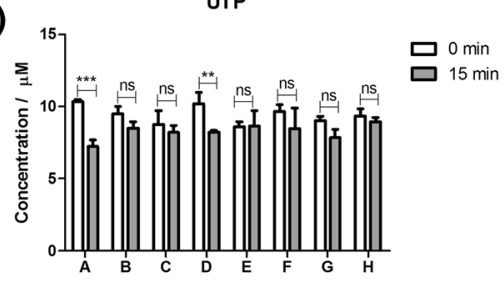

UDP

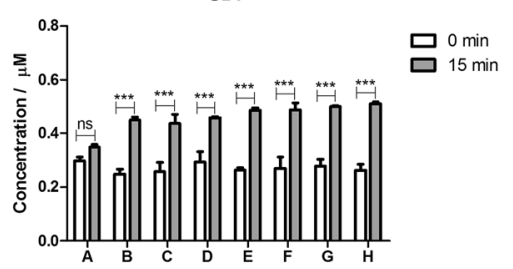

UMP

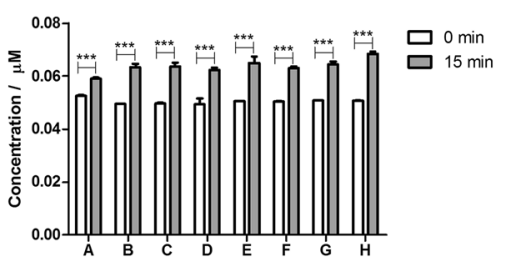

(D)

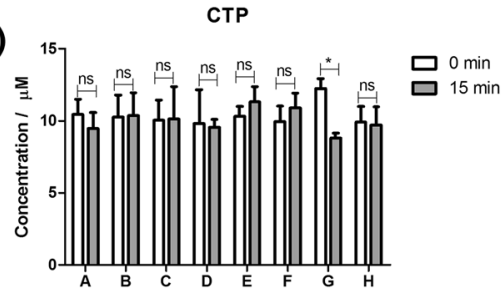

CDP

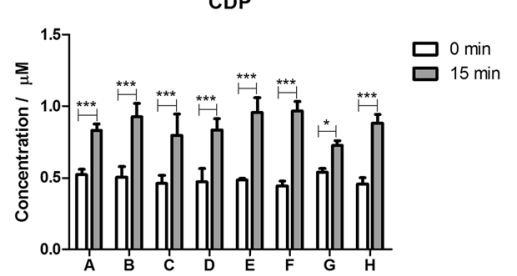

CMP

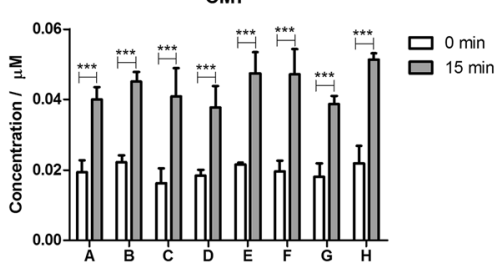

Fig. 5 Effect of specific groups of central carbon metabolites on the degradation of nucleotide triphosphates. a ATP, b GTP, c UTP, and d CTP were incubated with $4 \mu \mathrm{L}$ of solutions A-H (Table 1) and extracted with $75 \%$ (aq.) boiling ethanol. Each bar represents the mean of four independently prepared samples $( \pm S D)$. ANOVA: $* p<0.05 ; * * p<0.01 ; * * * p<0.001$ and $n s$ not significant 
concentration of nucleotide monophosphates ranged from $4 \%$ in solution $\mathrm{H}$ to $81 \%$ in solution $\mathrm{C}$ for AMP, from $38 \%$ in solution B to $88 \%$ in solution D for GMP, from $12 \%$ in solution A to $34 \%$ in solution $\mathrm{H}$ for UMP, and from $103 \%$ in solution B to $150 \%$ in solution C for CMP (Fig. 5). These results contrast with the observation that the yeast matrix appears to stabilize the phosphoric acid anhydride bond against hydrolysis, as certain metabolites and components of solutions A-H actually promoted hydrolysis.

A number of observations indicated that we are not only dealing with the interconversion of triphosphates into diand monophosphates. For example, there is a significant decrease in the concentration of ATP in solution E, which is not reflected in the much smaller increase in ADP (Fig. 5a). This indicates that while ATP is converted to ADP, there is a loss of ATP that cannot be accounted for. Another example is when ATP is incubated in solution D. While no reduction in ATP concentration was observed, there is a significant increase in ADP indicating that there is another source of ADP that is not derived from ATP. The increase in ADP (+272\%) in solution D may be explained by the presence of $\mathrm{NADP}^{+}$, which according to Hofmann et al. (2010), can be thermally degraded into ADP. A similar thermal degradation mechanism may explain the increase of $155 \%$ in ADP in solution A, which contains NADPH (Fig. 5a). It is also noteworthy that the concentration of AMP, which did not change significantly $(p>0.05$; ANOVA) in solutions $\mathrm{A}, \mathrm{D}, \mathrm{E}, \mathrm{F}, \mathrm{G}$ and $\mathrm{H}$, was significantly elevated in solutions $\mathrm{B}$ and $\mathrm{C}(p<0.001$; ANOVA) even prior to the extraction process $(\mathrm{t}=0 \mathrm{~min}$; Fig. 5a). Degradation of NADH and $\mathrm{NAD}^{+}$can produce AMP without ADP as an intermediate (Hofmann et al. 2010), which may explain the increase in AMP concentration prior to extraction. Furthermore, the presence of FAD might also contribute to this phenomenon.

These results show that the interconversion of energy metabolites is complex and cannot be solely addressed using stable isotope-dilution mass spectrometry. Gaining a better mechanistic understanding of the interconversion reactions under conditions of metabolite extraction is thus critical to obtain accurate results.

\section{Conclusions}

We studied nucleotide triphosphates as representatives of energy metabolites under boiling ethanol conditions. The major findings were that degradation of nucleotide triphosphates into nucleotide di- and monophosphates is prevented by the yeast matrix as compared to standard solutions and that the interconversion of structurally-related metabolites such as NADPH and $\mathrm{NADP}^{+}$into ADP and $\mathrm{NADH}, \mathrm{NAD}^{+}$or FAD into AMP may lead to erroneously elevated values that cannot be corrected for by the widely used stable-isotope-dilution mass spectrometry approach. To explain the matrix stabilization phenomenon, the influence of the culture medium (e.g. carbon source, vitamins, macro and micro elements) and particular groups of central carbon metabolites (amino acids, organic acids, sugar phosphates, coenzymes, and other nucleotides) was tested. While the basis of the observed stabilizing effect of the yeast matrix and possibly other cellular matrices remains unclear, we recommend studying the interconversion of metabolites in pilot experiments prior to largescale metabolomics.

Acknowledgements Marcel de Vries (University Medical Center Groningen, Department of Pediatrics) is acknowledged for help with operating the Synapt G2-Si mass spectrometer. The authors are grateful to Matthias Heinemann (University of Groningen, Molecular Systems Biology) for supporting the study with the ${ }^{13} \mathrm{C}$-labeled yeast cellular matrix.

Funding Andres Gil was funded by the Science, Technology and Innovation Department from Colombia-COLCIENCIAS (Grant: 6171-71294025). Dirk-Jan Reijngoud was supported by a Grant from the University Medical Center Groningen.

\section{Compliance with ethical standards}

Conflict of interest Andres Gil, David Siegel, Silke Bonsing-Vedelaar, Hjalmar Permentier, Dirk-Jan Reijngoud, Frank Dekker and Rainer Bischoff declare that they have no conflict of interest.

Research involving human participants and/or animals This article does not contain any studies with human participants or animals performed by any of the authors.

Open Access This article is distributed under the terms of the Creative Commons Attribution 4.0 International License (http://crea tivecommons.org/licenses/by/4.0/), which permits unrestricted use, distribution, and reproduction in any medium, provided you give appropriate credit to the original author(s) and the source, provide a link to the Creative Commons license, and indicate if changes were made.

\section{References}

Airoldi, C., Tripodi, F., Guzzi, C., Nicastro, R., \& Coccetti, P. (2015). NMR analysis of budding yeast metabolomics: A rapid method for sample preparation. Molecular BioSystems, 11(2), 379-383. doi: $10.1039 / \mathrm{c} 4 \mathrm{mb} 00452 \mathrm{c}$.

Amsler, P. E., \& Sigel, H. (1976). Comparison of the metal-ionpromoted dephosphorylation of the $5^{\prime}$-triphosphates of adenosine, inosine, guanosine and cytidine by $\mathrm{Mn}^{2+}, \mathrm{Ni}^{2+}$ and $\mathrm{Zn}^{2+}$ in binary and ternary complexes. European Journal of Biochemistry/FEBS, 63(2), 569-581. doi:10.1111/j.1432-1033.1976. tb10261.x.

Buescher, J. M., Moco, S., Sauer, U., \& Zamboni, N. (2010). Ultrahigh performance liquid chromatography-tandem mass spectrometry method for fast and robust quantification of anionic and aromatic metabolites. Analytical Chemistry, 82, 4403-4412. doi:10.1021/ac100101d. 
Canelas, A. B., Ras, C., ten Pierick, A., van Dam, J. C., Heijnen, J. J., \& van Gulik, W. M. (2008). Leakage-free rapid quenching technique for yeast metabolomics. Metabolomics, 4(3), 226-239. doi:10.1007/s11306-008-0116-4.

Canelas, A. B., ten Pierick, A., Ras, C., Seifar, R. M., van Dam, J. C., van Gulik, W. M., et al. (2009). Quantitative evaluation of intracellular metabolite extraction techniques for yeast metabolomics. Analytical Chemistry, 81, 7379-7389. doi:10.1021/ ac900999t.

Coulier, L., Bas, R., Jespersen, S., Verheij, E., Van Der Werf, M. J., \& Hankemeier, T. (2006). Simultaneous quantitative analysis of metabolites using ion-pair liquid chromatography-electrospray ionization mass spectrometry. Analytical Chemistry, 78(18), 6573-6582. doi:10.1021/ac0607616.

Daniel, R. M., Van Eckert, R., Holden, J. F., Truter, J., \& Crowan, D. A. (2004). The stability of biomolecules and the implications for life at high temperatures. In W. S. D. Wilcock, E. F. DeLong, D. S. Kelley, J. A. Baross, \& S. C. Cary (Eds.), The subseafloor biosphere at Mid-Ocean Ridges (Vol. 144). Washington: American Geophysical Union. doi:10.1029/GM144.

Dunn, W. B., \& Winder, C. L. (2011). Sample preparation related to the intracellular metabolome of yeast: Methods for quenching, extraction, and metabolite quantitation. Methods in Enzymology, 500, 277-297. doi:10.1016/B978-0-12-385118-5.00015-3.

European Medicines Agency (EMA), Committee for Medicinal Products for Human Use. (2012). Guideline on bioanalytical method validation. London, UK. Retrieved August 15, 2016 from http://www.ema.europa.eu/docs/en_GB/document_library/ Scientific_guideline/2011/08/WC500109686.pdf.

Faijes, M., Mars, A. E., \& Smid, E. J. (2007). Comparison of quenching and extraction methodologies for metabolome analysis of Lactobacillus plantarum. Microbial Cell Factories, 6, 1-8. doi:10.1186/1475-2859-6-27.

Gil, A., Siegel, D., Permentier, H., Reijngoud, D.-J. J., Dekker, F., \& Bischoff, R. (2015). Stability of energy metabolites-An often overlooked issue in metabolomics studies: A review. Electrophoresis, 36(18), 2156-2169. doi:10.1002/elps.201500031.

Gonzalez, B., François, J., \& Renaud, M. (1997). A rapid and reliable method for metabolite extraction in yeast using boiling buffered ethanol. Yeast, 13, 1347-1356. doi:10.1002/(SICI)10970061(199711)13:14<1347:AID-YEA176>3.0.CO;2-O.

Hofmann, D., Wirtz, A., Santiago-Schübel, B., Disko, U., \& Pohl, M. (2010). Structure elucidation of the thermal degradation products of the nucleotide cofactors NADH and NADPH by nano-ESIFTICR-MS and HPLC-MS. Analytical and Bioanalytical Chemistry, 398, 2803-2811. doi:10.1007/s00216-010-4111-z.

León, Z., García-Cañaveras, J. C., Donato, M. T., \& Lahoz, A. (2013). Mammalian cell metabolomics: Experimental design and sample preparation. Electrophoresis, 34, 2762-2775. doi:10.1002/elps. 201200605.

Loret, M. O., Pedersen, L., \& François, J. (2007). Revised procedures for yeast metabolites extraction: Application to a glucose pulse to carbon-limited yeast cultures, which reveals a transient activation of the purine salvage pathway. Yeast, 24(1), 47-60. doi:10.1002/yea. 1435 .

Lu, W., Bennett, B. D., \& Rabinowitz, J. D. (2008). Analytical strategies for LC-MS-based targeted metabolomics. Journal of Chromatography B, 871, 236-242. doi:10.1016/j.jchromb.2008. 04.031 .
Paglia, G., Magnúsdóttir, M., Thorlacius, S., Sigurjónsson, Ó. E., Gudmundsson, S., Palsson, B., et al. (2012). Intracellular metabolite profiling of platelets: Evaluation of extraction processes and chromatographic strategies. Journal of Chromatography B, 898, 111-120. doi:10.1016/j.jchromb.2012.04. 026.

Seifar, R. M., Ras, C., van Dam, J. C., van Gulik, W. M., Heijnen, J. J., \& van Winden, W. A. (2009). Simultaneous quantification of free nucleotides in complex biological samples using ion pair reversed phase liquid chromatography isotope dilution tandem mass spectrometry. Analytical Biochemistry, 388, 213-219. doi:10.1016/j.ab.2009.02.025.

Sellick, C. A., Knight, D., Croxford, A. S., Maqsood, A. R., Stephens, G. M., Goodacre, R., et al. (2010). Evaluation of extraction processes for intracellular metabolite profiling of mammalian cells: Matching extraction approaches to cell type and metabolite targets. Metabolomics, 6, 427-438. doi:10.1007/s11306-0100216-9.

Siegel, D., Permentier, H., Reijngoud, D. J., \& Bischoff, R. (2014). Chemical and technical challenges in the analysis of central carbon metabolites by liquid-chromatography mass spectrometry. Journal of Chromatography B, 966, 21-33. doi:10.1016/j. jchromb.2013.11.022.

Sigel, H., \& Amsler, P. E. (1976). Hydrolysis of nucleoside phosphates. 6. On the mechanism of the metal ion promoted dephosphorylation of purine nucleoside $5^{\prime}$-triphosphates. Journal of the American Chemical Society, 98(23), 7390-7400. doi:10. 1021/ja00439a047.

Sigel, H., \& Hofstetter, F. (1983). Metal-ion-promoted dephosphorylation of the 5 -triphosphates of uridine and thymidine, and a comparison with the reactivity in the corresponding cytidine and adenosine nucleotide systems. European Journal of Biochemistry/FEBS, 132(3), 569-577. doi:10.1111/j.1432-1033.1983. tb07401.x.

U.S. Department of Health and Human Services, Food and Drug Administration (HSS-FDA). (2013). Guidance for industrybioanalytical method validation. Rockville, USA. Retrieved August 18, 2016 from http://www.fda.gov/downloads/drugs/ guidancecomplianceregulatoryinformation/guidances/ucm 3681 07.pdf.

Verduyn, C., Postma, E., Scheffers, W. A., \& Van Dijken, J. P. (1992). Effect of benzoic acid on metabolic fluxes in yeasts: A continuous-culture study on the regulation of respiration and alcoholic fermentation. Yeast, 8(7), 501-517. doi:10.1002/yea. 320080703.

Villas-Bôas, S. G., \& Bruheim, P. (2007). Cold glycerol-saline: The promising quenching solution for accurate intracellular metabolite analysis of microbial cells. Analytical Biochemistry, 370, 87-97. doi:10.1016/j.ab.2007.06.028.

Vuckovic, D. (2012). Current trends and challenges in sample preparation for global metabolomics using liquid chromatography-mass spectrometry. Analytical and Bioanalytical Chemistry, 403, 1523-1548. doi:10.1007/s00216-012-6039-y.

Wu, L., Mashego, M. R., Van Dam, J. C., Proell, A. M., Vinke, J. L., Ras, C., et al. (2005). Quantitative analysis of the microbial metabolome by isotope dilution mass spectrometry using uniformly 13C-labeled cell extracts as internal standards. Analytical Biochemistry, 336(2), 164-171. doi:10.1016/j.ab. 2004.09.001. 